\title{
Total Power Minimization in Glitch-Free CMOS Circuits Considering Process Variation
}

\author{
Yuanlin $\mathrm{Lu}^{*}$ \\ Intel Corporation \\ Folsom, CA 95630, USA \\ yuanlin.lu@intel.com
}

\author{
Vishwani D. Agrawal \\ Auburn University \\ Auburn, AL 36849, USA \\ vagrawal@eng.auburn.edu
}

\begin{abstract}
Compared to subthreshold leakage, dynamic power is normally much less sensitive to the process variation due to its approximately linear relation to the process parameters. However, the average dynamic power of a circuit optimized by deterministic glitch elimination (using hazard filtering and path balancing) increases because glitches randomly start reappearing under the influence of process variation. Combining existing techniques, we propose a new statistical mixed integer linear programming (MILP) formulation, which combines glitch elimination and dual-threshold design to statistically minimize the total power in a glitch-free circuit under process variation.
\end{abstract}

\section{Introduction}

With the continuous increase of the density and performance of integrated circuits due to the scaling down of the CMOS technology, reducing power dissipation becomes a serious problem that every circuit designer has to face. At the same time, the increase in variability of several key process parameters can significantly affect the design and optimization of low power circuits in the nanometer regime [1-3]. Due to the exponential relation of leakage current with some process parameters, such as the effective gate length, oxide thickness and doping concentration, process variations can cause a significant increase in the leakage current. To minimize the effect of process variation, some techniques [1-3] statistically optimize the leakage power and circuit performance by dual- $V_{t h}$ assignment. Leakage current and delay are treated as random variables. A dynamic programming approach for leakage optimization by dual$V_{t h}$ assignment has been proposed [2] using two pruning criteria that stochastically identify pareto-optimal solutions and prune the sub-optimal ones. Another approach [1] solves the statistical leakage minimization problem by a theoretically rigorous formulation for dual$V_{t h}$ assignment and gate sizing.

Glitches are unnecessary signal transitions that account for $20 \%-70 \%$ of the dynamic switching power [4]. To eliminate glitches, we combine the techniques of

*Formerly with Department of Electrical and Computer Engineering, Auburn University, Auburn, AL 36849, USA. hazard filtering [5, 8-12] and path balancing $[6,8,11]$, referred to in this paper as glitch elimination. Compared to leakage power, dynamic power is normally much less sensitive to the process variation because of its approximately linear dependency on the process parameters. However, any deterministic glitch elimination technique becomes less effective under process variation, since the perfect hazard filtering conditions can be easily corrupted even with a small variation in some process parameters. Hu and Agrawal [13-14] proposed a technique to eliminate glitches under process variation. However, performance is sacrificed to obtain a process-variationresistant circuit, and the effect of process variation on leakage power is not considered.

Our work is motivated by the above research. To minimize the leakage power, we use a mixed integer linear programming (MILP) model to determine the optimal assignment of $V_{\text {th }}$ while controlling any reduction in performance. To eliminate the glitch power, additional MILP constraints determine the positions and values of the delay elements to be inserted to balance path delays. Statistical delay and leakage models are further adopted to reduce the total power in glitch-free circuits considering process variation.

\section{Background}

Lu and Agrawal [17] propose a statistical MILP formulation to minimize the impact of process variation on the subthreshold leakage. In this section, we extend that discussion to study the impact of process variation on dynamic power. Dynamic power comprises of two parts, logic switching power and glitch power:

$$
\begin{aligned}
P_{d y n} & =\frac{1}{2} C_{L} V^{2} \cdot A \cdot F \\
& =\text { Logic switching power }+ \text { Glitch power }
\end{aligned}
$$

where $A$ is switching activity and $F$ is the circuit operating frequency.

Logic switching power is directly proportional to the loading capacitances, $\mathrm{C}_{\mathrm{L}}$, which linearly depends upon gate sizes, gate width and gate length. Local (intra-die) process variation causes gate sizes to vary randomly and hence does not affect logic switching power too much. Global (inter-die) process variation changes gate sizes in similar ways and does vary the logic switching power. This 
also does not affect the solution of our MILP formulation, since gate delays and gate sizes in the MILP constraints either increase or decrease by the same percentage when global process variation is considered, and $T_{\max }$ (critical path delay that affects the circuit performance) is assumed to change accordingly [15].

The impact of process variation on glitch power is different and more complicated. Glitches are generated if the glitch filtering condition (2) [6] is not satisfied for cell $i$. Since inertial gate delays $d_{i}$ vary with process variations, inequality (2) may not be satisfied.

$$
d_{i}>T_{i}-t_{i}
$$

Where $T_{i}-t_{i}$ is the differential path delay at gate $i$. We consider the impact of global process variation and local process variation on glitch power, separately.

\section{- Impact of global process variation on glitches}

For every gate $i$, the timing window $T_{i}-t_{i}$ is actually determined by two timing paths, the fastest path (FPath) and the slowest path (SPath) from primary inputs to gate i. $T_{i}$ is the cumulative inertial gate delay along the slowest path, and $t_{i}$ is the cumulative inertial gate delay along that fastest path. Thus,

$$
T_{i}-t_{i}=\sum_{m \in S P a t h} d_{m}-\sum_{n \in F P a t h} d_{n}
$$

Assuming that there is $r \cdot 100 \%(r: 0 \sim 1)$ of global variation applied to the circuit, glitch filtering condition (2) for gate $i$ remains unchanged since both timing window, $T_{i}-t_{i}$, and gate delay vary by $r \cdot 100 \%$. Therefore, the technique of glitch elimination is resistant to the global process variation.

\section{- Impact of local process variation on glitches}

Let us consider the impact of local process variation on glitch elimination. When local variations occur in a circuit, $T_{i}$ and $t_{i}$ are the sum of gate delays, which vary randomly, along the slowest and fastest paths from primary inputs to cell $i$ 's inputs, so, $T_{i}-t_{i}$ is not very sensitive to process variations, while $d_{i}$ does change with the process variation. Therefore, it is very possible that the original glitch filtering conditions (2) can not be satisfied in the presence of local process variation.

As shown in Figure 1, there are three possible glitch filtering conditions. Both Figures 1(b) and 1(c) are glitch free while Figure 1(a) has a glitch. In an un-optimized circuit (with glitches), Figures 1(a) or 1(b) is represents a much more common condition for a gate. Although the condition of Figure 1(c) is still possible it has lower possibility. On the contrary, in an optimized glitch-free circuit, Figure 1(c) applies to many gates because Figure 1(a) is always forced to become Figure 1(c) by path balancing for glitch elimination.
With local process variation, Figures 2(a) and 2(b) show that the original condition is not so easily corrupted if only the variation of the timing window or the gate delay falls into the shaded areas, while Figure 2(c) is extremely sensitive to the local process variation, since a slight increase in the timing window or decrease in the gate delay can simply let an original glitch-free gate generate glitches at its output.

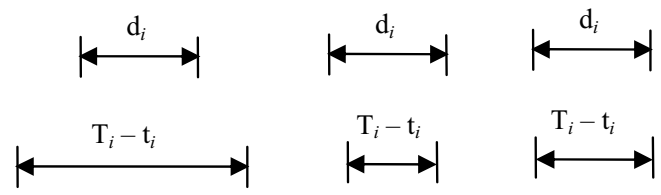

(a) Glitches generated (b) Glitch-free (c) Glitch-free

Figure 1. Three possible glitch elimination conditions.

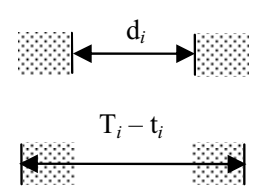

(a) Glitches generated
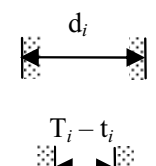

(b) Glitch-free
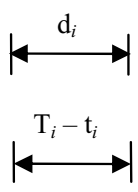

(c) Glitch-free
Figure 2. Three glitch elimination conditions under local process variation.

This explains why the dynamic power of an un-optimized circuit is much more resistant to local process variation than that of an optimized glitch-free circuit. The glitch elimination condition shown in Figure 1(c) cannot be really satisfied even with quite small process variation.

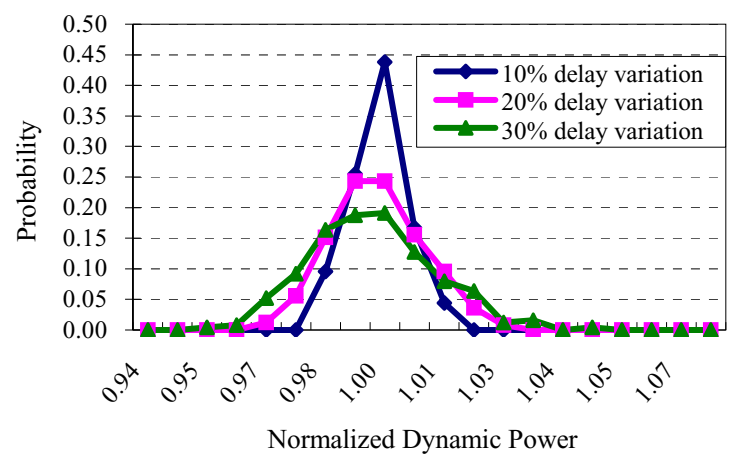

Figure 3. Normalized dynamic power distribution of unoptimized (with -glitches) C432 under local delay variation.

Figure 3 demonstrates the resistance of un-optimized circuits to the local process variation. We applied $10 \%$, $20 \%$ and $30 \%$ local delay variations, as may be caused by variations in gate-length-independent $V_{t h}$, to an unoptimized (with-glitch) version of circuit C432. The largest percentage of the mean value deviated from the nominal 
value is $0.22 \%$ and the maximum spread, $3 \times$ standard deviation / mean, is only $4.5 \%$.

The sensitivity of optimized glitch-free circuits to local process variation is illustrated in Figure 4. Both mean value and standard deviation of dynamic power distribution increase significantly with the increase of the local process variation. When $30 \%$ local variation was applied to the optimized glitch-free $\mathrm{C} 432$, its average dynamic power increased by $32 \%$ and almost became equal to the normalized dynamic power (1.34) of the unoptimized C432. In Figure 4, some samples of optimized C432 have dynamic power even larger than 1.34. We also note that every sample in Figure 4 consumes more than the nominal value, 1 , which is the expected minimumnormalized-dynamic-power of the optimized glitch-free C432. Process variation causes some glitches to be generated in the glitch-free circuit and hence increases the dynamic power.

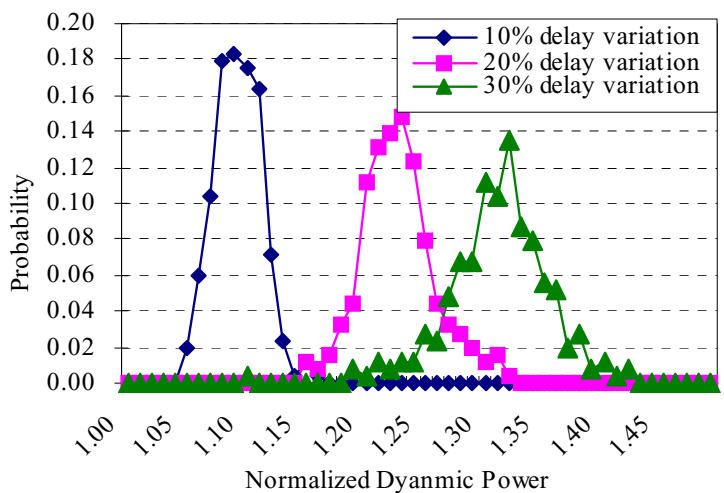

Figure 4. Normalized dynamic power distribution of optimized (glitch-free) C432 under local delay variation.

It is remarkable that the advantage of glitch elimination is totally lost due to the local process variation. Hence, the deterministic approach of glitch elimination is not useful for power optimization with process variation. In the following section, we combine the MILP formulations introduced in [15-17], and thus a new statistical MILP formulation is proposed to optimize total power under process variation and to fully utilize the advantage of the glitch elimination procedure.

The deterministic MILP [15-16] using glitch elimination and dual- $V_{\text {th }}$ assignment to reduce the total power consumption is a prerequisite procedure, which is modified to consider process variation.

\section{Statistical MILP for Total Power Optimization with Process Variation}

In the statistical MILP formulation, we treat all gate delays and timing window variables as random variables with normal distribution whose standard deviation is $\sigma_{\mathrm{r}}$.

\subsection{Variables}

\section{- Integer variables:}

In our cell library, each standard cell has two possible threshold voltages and three alternative sizes (1X, $2 \mathrm{X}$ and $4 \mathrm{X})$. Therefore, this MILP has six integer variables to allow alternative choices. The variables are denoted as,

\section{$X 1 L[i], X 2 L[i], X 4 L[i], X 1 H[i], X 2 H[i], X 4 H[i]$}

\section{- Continuous Variables:}

$\delta[i]$ - relaxed variable for the glitch filtering constraint of cell $i$. It will be discussed in Section 3.3.

Size[i] - size of cell $i$.

$I_{\text {leak }}[i]$ - nominal value of leakage of cell $i$.

$u_{-} D[i]$ - mean of inertial gate delay of cell $i$.

$s_{-} D[i]$ - standard deviation of inertial gate delay.

$u \_T[i]$ - mean of $T[i]$.

$s_{-} T[i]$ - standard deviation of $T[i]$.

$u \_t[i]$ - mean of $t[i]$.

$s \_t[i]$ - standard deviation of $t[i]$.

$u \_\Delta d[i, j]-$ mean of $\Delta d[i, j]$ (the delay of the inserted delay element).

$s \_\Delta d[i, j]$ - standard deviation of $\Delta d[i, j]$.

\subsection{Constants}

$\sigma_{r}$ - standard deviation of the process parameter variations.

$T_{\max }$ - the maximum expected circuit performance.

$S_{X 2}[i]$ - gate size of cell $i$ with $2 \mathrm{X}$ driving strength.

$W_{1}, W_{2}, W_{3}$ - weight factors.

$I_{X 2 L}[i], I_{X 2 H}[i]$ - nominal values of the subthrehold leakage of cell $i$ with $2 \mathrm{X}$ driving strength.

$D_{X 1 L}[i], \mathrm{D}_{\mathrm{X} 2 \mathrm{~L}}[i], D_{X 4 L}[i], D_{X 1 H}[i], D_{X 2 H}[i], D_{X 4 H}[i]$ nominal values of the inertial gate delay of cell $i$ at all six corners.

\subsection{Constraints}

\section{- Basic constraints}

Let LP solver choose one and only one optimal version for cell $i$.

$$
X 1 L[i]+X 2 L[i]+X 4 L[i]+X 1 H[i]+X 2 H[i]+X 4 H[i]=1
$$

Nominal value of the subthreshold leakage of cell $i$ :

$$
\begin{aligned}
I_{\text {leak }}[i]= & (0.5 \cdot X 1 L[i]+X 2 L[i]+2 \cdot X 4 L[i]) \cdot I_{X 2 L}[i]+ \\
& (0.5 \cdot X 1 H[i]+X 2 H[i]+2 \cdot X 4 H[i]) \cdot I_{X 2 H}[i]
\end{aligned}
$$

Mean and standard deviation of the gate delay of cell $i$ : 


$$
\begin{aligned}
u_{-} D[i]= & D_{X 1 L}[i] \cdot X 1 L[i]+D_{X 2 L}[i] \cdot X 2 L[i]+D_{X 4 L}[i] \cdot X 4 L[i]+ \\
& D_{X 2 L}[i] \cdot X 1 H[i]+D_{X 2 L}[i] \cdot X 2 H[i]+D_{X 4 L}[i] \cdot X 4 H[i]
\end{aligned}
$$

$s_{-} D[i]=\sigma_{r} \cdot u_{-} D[i]$

The size of cell $i$ :

$$
\text { Size }[i]=\left\{\begin{array}{l}
0.5 \cdot(X 1 L[i]+X 1 H[i])+(X 2 L[i]+X 2 H[i])+ \\
2 \cdot(X 4 L[i]+X 4 H[i])
\end{array}\right\} \cdot S_{X 2}[i]
$$

\section{- For glitch elimination}

Instead of using inequality (2), in the statistical method, we adopt the following glitch filtering constraint:

$$
u_{-} D[i]-3 \times s_{-} D[i] \geq\left(u_{-} T[i]+3 \times s_{-} T[i]\right)-\left(u_{-} t[i]-3 \times s_{-} t[i]\right)
$$

This constraint can leave certain margin for process variation in advance as shown in Figure 2(b) instead of Figure 2(c). However, normally the above worst case constraint is too tight to make CPLEX LP solver find a feasible solution. So, we add one nonnegative relaxed variable $\delta[i]$ to each glitch filtering constraint (9).

$\delta[i]+\left(u_{-} D[i]-3 \times s_{-} D[i]\right) \geq\left(u_{-} T[i]+3 \times s_{-} T[i]\right)-\left(u_{-} t[i]-3 \times s_{-} t[i]\right)$

In the objective function, by minimizing $\Sigma \delta[i]$, CPLEX LP solver will try to find one optimal solution to make as many of the constraints (10) satisfied as possible with a zero $\delta[i]$, which means the glitches of corresponding cells can be truly eliminated even in the worst case condition of process variation. Those constraints only being satisfied with the help of a positive $\delta[i]$ quite likely fail to filter glitches.

\section{- For maximal performance}

To keep the maximal performance, at every primary output $k$, let,

$$
u_{-} T[k]+3 \times s_{-} T[k] \leq T_{\max } \text {. }
$$

\subsection{Objective function}

The objective function minimizes the impact of process variation on the total power consumption:

Min \{the impact of process variation on the total power consumption

$=$ Min $\{$ mean and standard deviation of leakage power + mean and standard deviation of dynamic power

$=\operatorname{Min}\left\{\begin{array}{l}W_{1} \cdot C_{1} \sum_{i} I_{\text {leak }}[i]+ \\ W_{2} \cdot\left(C_{2} \sum_{i} \text { size }[i]+C_{3} \sum_{i} \sum_{j} \mu_{-} \Delta d[i, j]\right)+ \\ W_{3} \cdot \sum_{i} \delta[i]\end{array}\right\}$
$C_{1}, C_{2}$ and $C_{3}$ are fitting parameters to let three terms $\left(C_{1} \Sigma I_{\text {leak }}[i], C_{2} \Sigma\right.$ size $[i]$ and $\left.C_{3} \Sigma \Sigma u_{-} \Delta d[i, j]\right)$ have the same units $(\mu \mathrm{W})$.

The impact of process variation on both mean and standard deviation of the power consumption should be considered. For leakage, a smaller mean value automatically implies a narrower spread of leakage power distribution since more gates are assigned high $V_{t h}$. $\operatorname{Min}\left(C_{l} \Sigma I_{\text {leak }}[i]\right)$ should be enough to minimize the impact of process variation on the total subthreshold leakage. For the dynamic power, standard deviation of the dynamic power distribution is determined by $\Sigma \delta[i]$, and $\left(C_{2} \Sigma\right.$ size $\left.[i]+C_{3} \Sigma \Sigma u_{-} \Delta d[i, j]\right)$ affects the average dynamic power. Therefore, we should minimize $\quad\left(C_{2} \Sigma\right.$ size $\left.[i]+C_{3} \Sigma \Sigma \quad u_{-} \Delta d[i, j]\right)$ and $\Sigma \delta[i]$, simultaneously.

The objective function (12) is composed of three parts (three single objectives), namely, minimize the average leakage power, minimize the average dynamic power and minimize the standard deviation of the dynamic power. It is a multi-objective function in which individual objectives conflict. For instance, minimization of $\Sigma \delta[i]$ results in an increase of $\Sigma \Sigma u_{-} \Delta d[i, j]$, and optimization of $\Sigma I_{\text {leak }}[i]$ leads to a larger $\Sigma$ size $[i]$, etc. It is not easy to get one optimum value for every single objective. What we can do instinctively is to carefully select weight factors, $W_{1}, W_{2}$ and $W_{3}$ to make a tradeoff among the three objectives.

It should be noticed that the solution provided by a deterministic MILP [15-16] gives us a rough idea of which one is the dominant component between leakage and dynamic power. We also get their exact optimal values (power consumption) for the optimized circuit. Based on that information, we can choose weight factors and add some empirical constraints on the largest allowable minimal leakage or dynamic power in the statistical MILP formulation.

The choice of minimizing the impact of process variation either on leakage or on dynamic power depends on which one is the dominant power consumer, and the circuit applications as well. For a circuit optimized by the deterministic MILP, we consider:

- Case 1 - if the optimal leakage is much less than the optimal dynamic power and its large spread due to process variation (for example, $5 \mathrm{X}$ difference under $30 \%$ global process variation ) can still be ignored, we need put much more emphasis on dynamic power changes being resistant to process variation;

- Case 2 - if the optimal leakage is comparable to the optimal dynamic power, and most of the time the circuit remains in standby mode( for example, circuits of cell phones) the impact of process variation on the optimal leakage should be minimized with priority since leakage is much more sensitive to the process variation;

- Case 3 - if the optimal leakage is comparable to the optimal dynamic power, and most of the time the 
circuit is in the active mode (for example, circuits of portable GPS, portable game machines, etc.) both the mean and standard deviation of the dynamic power distribution should be optimized.

\subsection{Minimizing impact of process variation on leakage}

In case 1 and case 3 , dynamic power is the dominant component of the total power consumption. Its standard deviation is determined by the number of glitch filtering constraints (10) whose $\delta[i]$ have positive values. So, in the MILP objective function (13), we first let $W 3$ be infinitely large to put the highest priority on minimizing $\Sigma \delta[i]:$

$$
\operatorname{Min}\left\{W 1 \cdot \sum_{i} I_{\text {leak }}[i]+W 2 \cdot\left(\sum_{i} \operatorname{size}[i]+\sum_{i} \sum_{j} \Delta d[i, j]\right)+\underset{W 3->\infty}{W} \sum_{i} \delta[i]\right\}
$$

Although MILP tries to minimize $\Sigma \delta[i], \delta[i]$ for some gates may still be positive since the constraint (9) is too tight to be satisfied without the help of a positive $\delta[i]$. Every positive $\delta[i]$ possibly results in the glitch generation at gate $i$ 's output. From Figure 4, we also see that the average dynamic power almost linearly increases with the process variation. This increase is contributed by the glitches caused by the process variatio. To counteract the increase in the average dynamic power due to those glitches, or to let the really average dynamic power in process variation condition still be close to that achieved by the deterministic MILP formulation, we sacrifice some leakage power and get a smaller logic switching power. This can be achieved by letting $W 1$ and $W 2$ both equal to 1 in the MILP objective function (14) and adding a new constraint (15) to the statistical MILP formation.

$$
\operatorname{Min}\left\{C_{1} \sum_{i} I_{\text {leak }}[i]+\left(C_{2} \sum_{i} \operatorname{size}[i]+C_{3} \sum_{i} \sum_{j} \Delta d[i, j]\right)+\underset{W \rightarrow \infty}{W 3} \sum_{i} \delta[i]\right\}
$$

$$
C_{2} \sum_{i} \operatorname{size}[i]+C_{3} \sum_{i} \sum_{j} \Delta d[i, j]<P_{d y n_{-} o p t} / \rho \quad(\rho>1)
$$

$P_{\text {dyn_opt }}$ is the optimal dynamic power obtained by the deterministic MILP [15-16] and $\rho$ is a constant determined by the process variation. By letting $\rho$ larger than 1, the statistical MILP formulation can give an optimal circuit with less dynamic power.

\subsection{Minimizing impact of process variation on leakage}

In case 2, leakage almost equals or is even larger than the dynamic power. Since leakage is so sensitive to the process variation that we cannot minimize the effect of process variation on the dynamic power by sacrificing leakage any more. The technique of eliminating glitches has to be discarded since the increase in the average dynamic power under process variation may be close to or even larger than the glitch power saved. To make the leakage of optimized circuits resistant to the process variation, we can still use the MILP proposed in [17] except every gate has six possible choices instead of two choices.

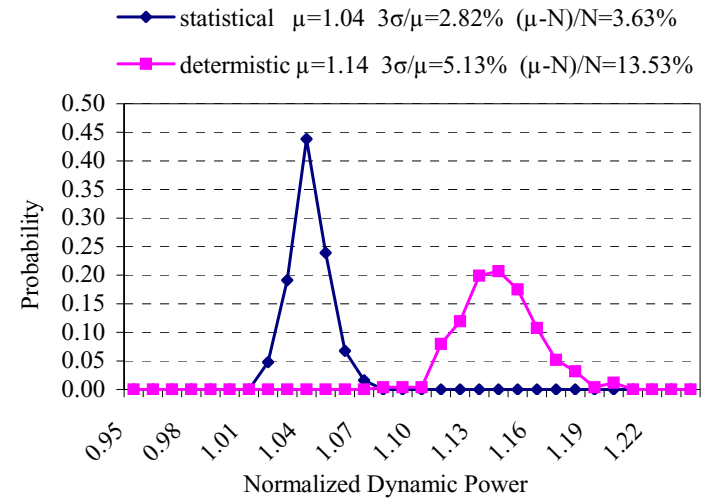

Figure 5. Comparison of the impacts of $15 \%$ local process variation on the dynamic power in $\mathrm{C} 432$ which is optimized by the statistical MILP with the emphasis on the resistance of dynamic power to process variation, or by the deterministic MILP [15-16]. (Dynamic power $=1$ is the expected normalized minimum dynamic power in the optimized glitch-free C432).

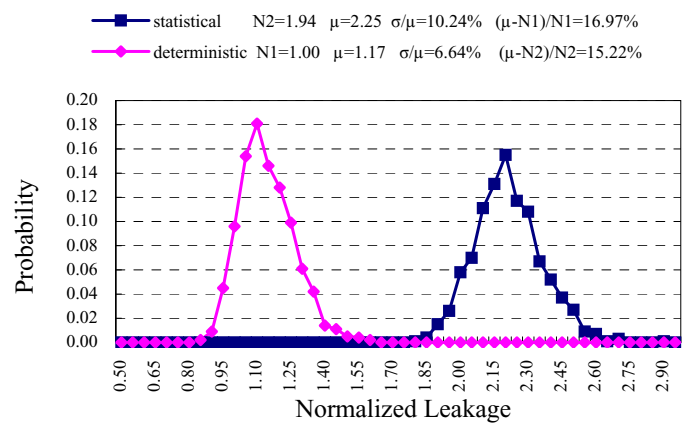

Figure 6. Comparison of the impacts of $15 \%$ local $L_{\text {eff }}$ process variation on the leakage power in $\mathrm{C} 432$ which are optimized by the statistical MILP with the emphasis on the resistance of dynamic power to process variation, or the deterministic MILP [15-16]. (N1 and N2 are the normalized nominal leakage power in the optimized glitchfree C432).

\section{Results}

In C432 optimized by the deterministic MILP formulation [15-16], the optimized total power comprises $59.3 \mu \mathrm{W}$ dynamic power and $5.54 \mu \mathrm{W}$ leakage power. With $15 \%$ local process variation, the average dynamic power increases $13.53 \%$ with $5.13 \%$ standard deviation. To reduce the impact of process variation on the dynamic power, the objective function (14) and constraint (15) (let $P_{\text {dyn_opt }}=59.3 \mu \mathrm{W}$ and $\left.\rho=1.10\right)$ are adopted in the statistical MILP formulation. The two curves in Figure 5 show that 
the average dynamic power only increases $3.63 \%$ instead of $13.53 \%$, and standard deviation is also reduced to $2.82 \%$ from $5.13 \%$ when $15 \%$ local process variation is applied to the optimized glitch-free C432, although at a cost of $94 \%$ average leakage power increase (from 1.0 to 1.94 ) and a little bit wider spread of leakage power distribution, which is shown in Figure 6.

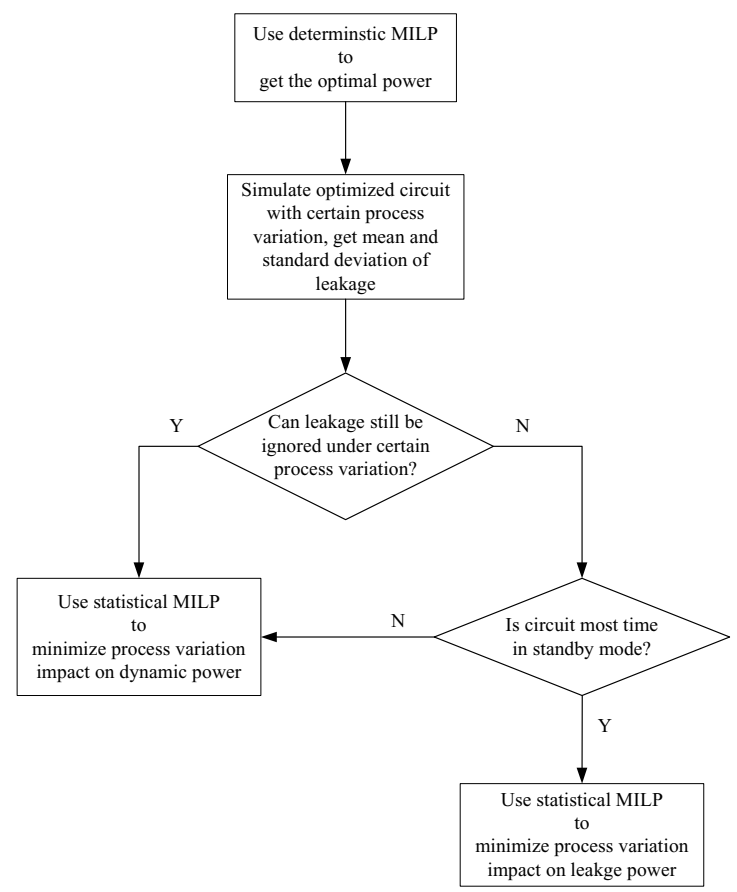

Figure 7. An algorithm to determine whether leakage or dynamic power should be optimized with process variation.

\section{Summary}

In this paper, the impact of process variation on dynamic power is analyzed, and a statistical MILP formulation is presented to minimize the total (dynamic and leakage) power in glitch-free circuits considering process variation. The impact of process variation on dynamic power can be minimized by giving up some leakage if the dynamic power is still the dominant power component under process variation. Figure 7 gives a flowchart of how to make a decision about which one, leakage or dynamic power, should be optimized with process variation.

\section{References}

[1] M. Mani, A. Devgan, and M. Orshansky, "An Efficient Algorithm for Statistical Minimization of Total Power Under Timing Yield Constraints," Proc. Design Automation Conference, 2005, pp. 309-314.
[2] A. Davoodi and A. Srivastava, "Probabilistic Dual- $V_{t h}$ Optimization Under Variability," Proc. ISLPED, 2005, pp. 143-147.

[3] A. Srivastava, D. Sylvester, D. Blaauw, "Statistical Optimization of Leakage Power Considering Process Variations Using Dual- $V_{t h}$ and Sizing," Proc. Design Automation Conf., 2004, pp. 773-778.

[4] A. P. Chandrakasan and R. W. Brodersen, Low Power Digital CMOS Design. Boston: Springer, 1995.

[5] V. D. Agrawal, "Low Power Design by Hazard Filtering," Proc. 10th Int. Conf. VLSI Design, 1997, pp. 193-197.

[6] V. D. Agrawal, M. L. Bushnell, G. Parthasarathy, and R. Ramadoss, "Digital Circuit Design for Minimum Transient Energy and a Linear Programming Method," Proc. 12th International Conf. VLSI Design, 1999, pp. 434-439.

[7] E. Jacobs and M. Berkelaar, "Using Gate Sizing to Reduce Glitch Power," Proc. PRORISC/IEEE Workshop on Circuits, Systems and Signal Processing, 1996, pp. 183-188.

[8] S. Kim, J. Kim, and S. Y. Hwang, "New Path Balancing Algorithm for Glitch Power Reduction," IEE Proc. Circuits, Devices and Systems, vol. 148, no. 3, pp. 151-156, 2001.

[9] C. V. Schimpfle, A. Wroblewski, and J. A. Nossek, "Transistor Sizing for Switching Activity Reduction in Digital Circuits," Proc. European Conference on Theory and Design, 1999, pp. 114-117.

[10] A. Wroblewski, C. V. Schimpfle, and J. A. Nossek, "Automated Transistor Sizing Algorithm for Minimizing Spurious Switching Activities in CMOS Circuits," Proc. IEEE International Symposium on Circuits and Systems, 2000, pp. 291-294.

[11] T. Raja, V. D. Agrawal, and M. L. Bushnell, "Minimum Dynamic Power CMOS Circuit Design by a Reduced Constraint Set Linear Program," Proc. 16th International Conf. VLSI Design, 2003, pp. 527-532.

[12] T. Raja, V. D. Agrawal, and M. L. Bushnell, "Variable Input Delay CMOS Logic for Low Power Design," Proc. 18th International Conf. VLSI Design, 2005, pp. 596-603.

[13] F. Hu and V. D. Agrawal, "Input-Specific Dynamic Power Optimization for VLSI Circuits," Proc. Int. Symp. Low Power Electronics and Design, 2006, pp. 232-237.

[14] F. Hu, "Process-Variation-Resistant Dynamic Power Optimization for VLSI Circuits," PhD Thesis, Auburn, Alabama: Auburn University, May 2006.

[15] Y. Lu and V. D. Agrawal, "CMOS Leakage and Glitch Power Minimization for Power-Performance Tradeoff," Journal of Low Power Electronics, vol. 2, no. 3, pp. 378387, Dec. 2006

[16] Y. Lu and V. D. Agrawal, "Leakage and Dynamic Glitch Power Minimization Using Integer Linear Programming for Vth Assignment and Path Balancing," Proc. of the International Workshop on Power and Timing Modeling, Optimization and Simulation, 2005, pp. 217-226.

[17] Y. Lu and V. D. Agrawal, "Statistical Leakage and Timing Optimization for Submicron Process Variation," Proc. 20th International Conf. VLSI Design, 2007, pp. 439-444. 